\title{
Intrinsic Brain Surface Conformal Mapping using a Variational Method
}

\author{
Yalin Wang ${ }^{a}$ and Xianfeng $\mathrm{Gu}^{b}$ and Tony F. Chan ${ }^{a}$ \\ and Paul M. Thompson ${ }^{c}$ and Shing-Tung Yau ${ }^{d}$ \\ ${ }^{a}$ Mathematics Department, UCLA Los Angeles, CA 90095, USA \\ ${ }^{b}$ CISE, University of Florida Gainesville, FL 32611, USA \\ ${ }^{c}$ LONI, UCLA School of Medicine Los Angeles, CA 90095, USA \\ ${ }^{d}$ Department of Mathematics, Harvard University Cambridge, MA 02138, USA
}

\begin{abstract}
We developed a general method for global conformal parameterizations based on the structure of the cohomology group of holomorphic one-forms with or without boundaries. ${ }^{1,2}$ For genus zero surfaces, our algorithm can find a unique mapping between any two genus zero manifolds by minimizing the harmonic energy of the map. In this paper, we apply the algorithm to the cortical surface matching problem. We use a mesh structure to represent the brain surface. Further constraints are added to ensure that the conformal map is unique. Empirical tests on MRI data show that the mappings preserve angular relationships, are stable in MRIs acquired at different times, and are robust to differences in data triangulation, and resolution. Compared with other brain surface conformal mapping algorithms, our algorithm is more stable and has good extensibility.
\end{abstract}

\section{INTRODUCTION}

Recent developments in brain imaging have accelerated the collection and databasing of brain maps. Nonetheless, computational problems arise when integrating and comparing brain data. One way to analyze and compare brain data is to map them into a canonical space while retaining geometric information on the original structures as far as possible. ${ }^{3-7}$

\subsection{Previous work}

Conformal surface parameterizations have been studied intensively. Most works on conformal parameterizations deal with surface patches homeomorphic to topological disks. For surfaces with arbitrary topologies, Gu and $Y_{\mathrm{au}}{ }^{1}$ introduce a general conformal parametrization based on a nonlinear flow for the genus zero case, and on the structure of cohomology group of holomorphic one form in the case of genus greater than one. They generalize the method for surfaces with boundaries in. ${ }^{2}$ In this paper, we apply part of these algorithms (for genus zero) to cortical surface matching problem and report our experimental results. In particular, the algorithms used in Section II, III, and IV, are from ${ }^{1,2}$ and the data compression using spherical harmonic was also conceived there for other purposes.

For genus zero surfaces, there are five basic approaches to achieve conformal parameterizations.

1. Harmonic energy minimization. Eck et al. ${ }^{8}$ introduce the discrete harmonic map, which approximates the continuous harmonic map ${ }^{9}$ by minimizing a metric dispersion criterion. Desbrun et al. ${ }^{10,11}$ compute the discrete Dirichlet energy and apply conformal parameterization to interactive geometry remeshing. Pinkall and Polthier compute the discrete harmonic map and Hodge star operator for the purpose of creating a minimal surface. ${ }^{12}$ Kanai et al. use a harmonic map for geometric metamorphosis in. ${ }^{13}$

Further author information: (Send correspondence to Yalin Wang or Xianfeng Gu)

Yalin Wang: E-mail: ylwang@math.ucla.edu, Telephone 13108258525

Xianfeng Gu: E-mail: gu@cise.ufl.edu, Telephone 13523921200

Tony C. Chan: E-mail: chan@math.ucla.edu, Telephone 13108252601

Paul M. Thompson: E-mail: thompson@loni.ucla.edu, Telephone 13102062101

Shing-Tung Yau: E-mail: yau@math.harvard.edu, Telephone 16174950836 
While discrete harmonic map were used, it is not clear that it approximates the harmonic map defined in the smooth category. Gu and Yau in ${ }^{1}$ introduce a non-linear optimization method to compute global conformal parameterizations for genus zero surfaces. The optimization is carried out in the tangent spaces of the sphere. It is different from the previous optimization methods. It computes global parametrizations for genus zero surfaces.

2. Cauchy-Riemann equation approximation. Levy et al. ${ }^{14}$ compute a quasi-conformal parameterization of topological disks by approximating the Cauchy-Riemann equation using the least squares method. They show rigorously that the quasi-conformal parameterization exists uniquely, and is invariant to similarity transformations, independent of resolution, and orientation preserving.

3. Laplacian operator linearization. Haker et al. ${ }^{5,15}$ use a method to compute a global conformal mapping from a genus zero surface to a sphere by representing the Laplace-Beltrami operator as a linear system.

4. Angle based method. Sheffer et al. ${ }^{16}$ introduce an angle based flattening method to flatten a mesh to a 2D plane so that it minimizes the relative distortion of the planar angles with respect to their counterparts in the three-dimensional space.

5. Circle packing. Circle packing is introduced in. ${ }^{4,17}$ Classical analytic functions can be approximated using circle packing. For general surfaces in $\mathbb{R}^{3}$, the circle packing method considers only the connectivity but not the geometry, so it is not suitable for our parameterization purpose.

\subsection{Basic Idea}

It is well known that any genus zero surface can be mapped conformally onto the sphere and any local portion thereof onto a disk. This mapping, a conformal equivalence, is one-to-one, onto, and angle-preserving. Moreover, the elements of the first fundamental form remain unchanged, except for a scaling factor (the so-called Conformal Factor). For this reason, conformal mappings are often described as being similarities in the small. Since the cortical surface of the brain is a genus zero surface, conformal mapping offers a convenient method to retain local geometric information, when mapping data between surfaces. We illustrate the conformal parameterization via the texture mapping of a checker board in Figure 1.

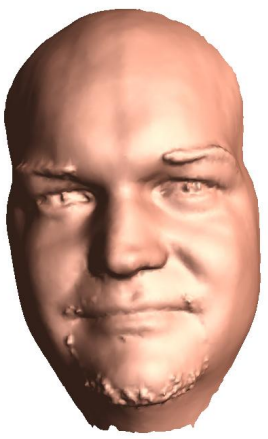

(a)

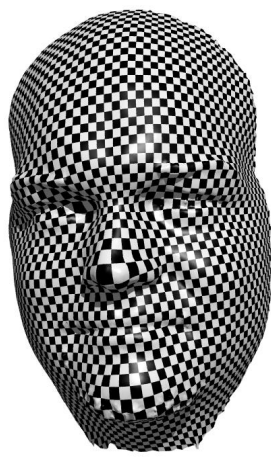

(b)

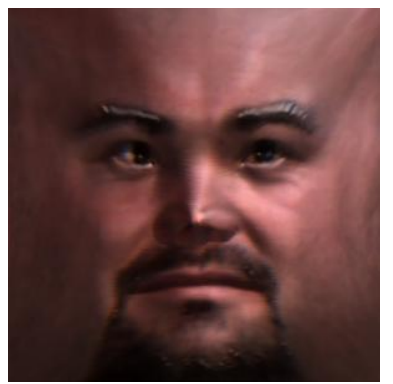

(c)

Figure 1. Conformal surface parameterization examples. (a) is a real male face. (c) is a square into which the human face is conformally mapped. (b) is the conformal parameterization illustrated by the texture map. As shown, the right angles on the checkboard are well preserved on the surface in (b).

Indeed, several groups have created flattened representations or visualizations of the cerebral cortex or cerebel$\operatorname{lum}^{4,5}$ using conformal mapping techniques. However, these approaches are either not strictly angle preserving, ${ }^{4}$ or there may be areas with large geometric distortions.$^{5}$ In this paper, we propose a new genus zero surface conformal mapping algorithm ${ }^{1}$ and demonstrate its use in computing conformal mappings between brain 
surfaces. Our algorithm depends only on the surface geometry and is invariant to changes in image resolution and the specifics of the data triangulation. Our experimental results show that our algorithm has advantageous properties for cortical surface matching.

Suppose $K$ is a simplicial complex, and $f:|K| \rightarrow R^{3}$, which embeds $|K|$ in $R^{3}$; then $(K, f)$ is called a mesh. Given two genus zero meshes $M_{1}, M_{2}$, there are many conformal mappings between them. Our algorithm for computing conformal mappings is based on the fact that for genus zero surfaces $S_{1}, S_{2}, f: S_{1} \rightarrow S_{2}$ is conformal if and only if $f$ is harmonic. All conformal mappings between $S_{1}, S_{2}$ form a group, the so-called Möbius group. Figure 2 show some examples of Möbius transformations. We can conformally map the surface of the head of Michelangelo's David to a sphere. When we draw the longitude and latitude lines on the sphere, we can induce correspond circles on the original surface (a) and (b). We apply a Möbius transformation to the sphere and make the two eyes become north and south poles. When we draw the longitude and latitude lines again (c), we get an interesting result shown in (d). Note all the right angles between the lines are well preserved in (b) and (d). This example demonstrates that all the conformal mapping results form a Möbius group.

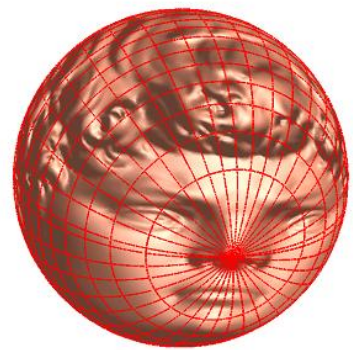

(a)

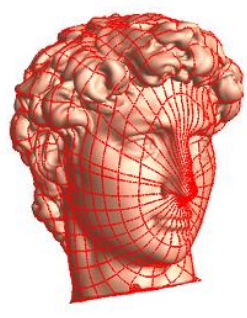

(b)

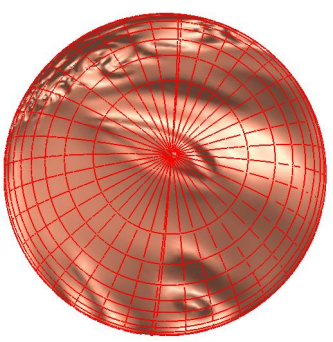

(c)

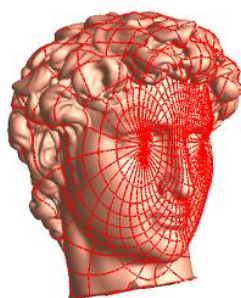

(d)

Figure 2. Möbius transformation example. We conformally map the surface of the head of Michelangelo's David to a sphere. In (a), we select the nose tip as the north pole and draw longitude lines and latitude lines on the sphere. (b) shows the results on the original David head model. We apply a Möbius transformation on the sphere in (a) and make the two eyes become the north and south poles. When drawing the longitude lines and latitude on the sphere (c), we get an interesting configuration for the lines on the original surface (d).

Our method is as follows: we first find a homeomorphism $h$ between $M_{1}$ and $M_{2}$, then deform $h$ such that $h$ minimizes the harmonic energy. To ensure the convergence of the algorithm, constraints are added; this also ensures that there is a unique conformal map.

This paper is organized as follows. In Section 2, we give the definitions of a piecewise linear function space, inner product and piecewise Laplacian. In Section 3, we describe the steepest descent algorithm which is used to minimize the string energy. In Section 4, we detail our conformal spherical mapping algorithms. Experimental results on conformal mapping for brain surfaces are reported in Section 7. We conclude the paper in Section 8.

\section{PIECEWISE LINEAR FUNCTION SPACE, INNER PRODUCT AND LAPLACIAN}

For the diffeomorphisms between genus zero surfaces, if the map minimizes the harmonic energy, then it is conformal. Based on this fact, the algorithm is designed as a steepest descent method.

This section formulates the mathematical concepts in a rigorous way. The major concepts, the harmonic energy of a map and its derivative, are defined. Because all the calculation is carried out on surfaces, we use the absolute derivative. Furthermore, for the purpose of implementation, we introduce the definitions in discrete form.

We use $K$ to represent the simplicial complex, $u, v$ to denote the vertices, and $\{u, v\}$ to denote the edge spanned by $u, v$. We use $f, g$ to represent the piecewise linear functions defined on $K$, use $\vec{f}$ to represent vector value functions. We use $\Delta_{P L}$ to represent the discrete Laplacian operator.

Definition 2.1. All piecewise linear functions defined on $K$ form a linear space, denoted by $C^{P L}(K)$. 
In practice, we use $C^{P L}(K)$ to approximate all functions defined on $K$. So the final result is an approximation to the conformal mapping. The higher the resolution of the mesh is, the more accurate the approximated conformal mapping is.

DEFINITION 2.2. \}labelinnerproduct Suppose a set of string constants $k_{u, v}$ are assigned for each edge $\{u, v\}$, the inner product on $C^{P L}$ is defined as the quadratic form

$$
<f, g>=\frac{1}{2} \sum_{\{u, v\} \in K} k_{u, v}(f(u)-f(v))(g(u)-g(v))
$$

The energy is defined as the norm on $C^{P L}$.

Definition 2.3. Suppose $f \in C^{P L}$, the string energy is defined as:

$$
E(f)=<f, f>=\sum_{\{u, v\} \in K} k_{u, v}\|f(u)-f(v)\|^{2}
$$

By changing the string constants $k_{u, v}$ in the energy formula, we can define different string energies.

DEFINITION 2.4. If string constants $k_{u, v} \equiv 1$, the string energy is known as the Tuette energy.

Definition 2.5. Suppose edge $\{u, v\}$ has two adjacent faces $T_{\alpha}, T_{\beta}$, with $T_{\alpha}=\left\{v_{0}, v_{1}, v_{2}\right\}$, define the parameters

$$
\begin{aligned}
a_{v_{1}, v_{2}}^{\alpha} & =\frac{1}{2} \frac{\left(v_{1}-v_{3}\right) \cdot\left(v_{2}-v_{3}\right)}{\left|\left(v_{1}-v_{3}\right) \times\left(v_{2}-v_{3}\right)\right|} \\
a_{v_{2}, v_{3}}^{\alpha} & =\frac{1}{2} \frac{\left(v_{2}-v_{1}\right) \cdot\left(v_{3}-v_{1}\right)}{\left|\left(v_{2}-v_{1}\right) \times\left(v_{3}-v_{1}\right)\right|} \\
a_{v_{3}, v_{1}}^{\alpha} & =\frac{1}{2} \frac{\left(v_{3}-v_{2}\right) \cdot\left(v_{1}-v_{2}\right)}{\left|\left(v_{3}-v_{2}\right) \times\left(v_{1}-v_{2}\right)\right|}
\end{aligned}
$$

$T_{\beta}$ is defined similarly. If $k_{u, v}=a_{u, v}^{\alpha}+a_{u, v}^{\beta}$, the string energy obtained is called the harmonic energy.

The string energy is always a quadratic form. By carefully choosing the string coefficients, we make sure the quadratic form is positive definite. This will guarantee the convergence of the steepest descent method.

Definition 2.6. The piecewise Laplacian is the linear operator $\Delta_{P L}: C^{P L} \rightarrow C^{P L}$ on the space of piecewise linear functions on $K$, defined by the formula

$$
\Delta_{P L}(f)=\sum_{\{u, v\} \in K} k_{u, v}(f(v)-f(u))
$$

If $f$ minimizes the string energy, then $f$ satisfies the condition $\Delta_{P L}(f)=0$. Suppose $M_{1}, M_{2}$ are two meshes and the map $\vec{f}: M_{1} \rightarrow M_{2}$ is a map between them, $\vec{f}$ can be treated as a map from $M_{1}$ to $R^{3}$ also.

Definition 2.7. For a map $\vec{f}: M_{1} \rightarrow R^{3}, \vec{f}=\left(f_{0}, f_{1}, f_{2}\right), f_{i} \in C^{P L}, i=0,1,2$, we define the energy as the norm of $\vec{f}$ :

$$
E(\vec{f})=\sum_{i=0}^{2} E\left(f_{i}\right)
$$

The Laplacian is defined in a similar way,

Definition 2.8. For a map $\vec{f}: M_{1} \rightarrow R^{3}$, the piecewise Laplacian of $\vec{f}$ is

$$
\Delta_{P L} \vec{f}=\left(\Delta_{P L} f_{0}, \Delta_{P L} f_{1}, \Delta_{P L} f_{2}\right)
$$


A map $\vec{f}: M_{1} \rightarrow M_{2}$ is harmonic, if and only if $\Delta_{P L} \vec{f}$ only has a normal component, and its tangential component is zero.

$$
\Delta_{P L}(\vec{f})=\left(\Delta_{P L} \vec{f}\right)^{\perp}
$$

\section{STEEPEST DESCENT ALGORITHM}

Suppose we would like to compute a mapping $\vec{f}: M_{1} \rightarrow M_{2}$ such that $\vec{f}$ minimizes a string energy $E(\vec{f})$. This can be solved easily by the steepest descent algorithm:

$$
\frac{d \vec{f}(t)}{d t}=-\Delta \vec{f}(t)
$$

$\vec{f}\left(M_{1}\right)$ is constrained to be on $M_{2}$, so $-\Delta \vec{f}$ is a section of $M_{2}$ 's tangent bundle.

Specifically, suppose $\vec{f}: M_{1} \rightarrow M_{2}$, and denote the image of each vertex $v \in K_{1}$ as $\vec{f}(v)$. The normal on $M_{2}$ at $\vec{f}(v)$ is $\vec{n}(\vec{f}(v))$. Define the normal component as

DeFinition 3.1. The normal component

$$
(\Delta \vec{f}(v))^{\perp}=<\Delta \vec{f}(v), \vec{n}(\vec{f}(v))>\vec{n}(\vec{f}(v)),
$$

where $<,>$ is the inner product in $R^{3}$.

Definition 3.2. The absolute derivative is defined as

$$
D \vec{f}(v)=\Delta \vec{f}(v)-(\Delta \vec{f}(v))^{\perp}
$$

Then equation (14) is $\delta \vec{f}=-D \vec{f} \times \delta t$.

\section{CONFORMAL SPHERICAL MAPPING}

Suppose $M_{2}$ is $S^{2}$, then a conformal mapping $\vec{f}: M_{1} \rightarrow S^{2}$ can be constructed by using the steepest descent method. The major difficulty is that the solution is not unique but forms a Möbius group.

Definition 4.1. Mapping $f: \mathcal{C} \rightarrow \mathcal{C}$ is a Möbius transformation if and only if

$$
f(z)=\frac{a z+b}{c z+d}, a, b, c, d \in \mathcal{C}, a d-b c \neq 0,
$$

where $\mathcal{C}$ is the complex plane.

All Möbius transformations form the Möbius transformation group. In order to determine a unique solution we can add different constraints. In practice we use the following two constraints: the zero mass-center constraint and a landmark constraint.

Definition 4.2. Mapping $\vec{f}: M_{1} \rightarrow M_{2}$ satisfies the zero mass-center condition if and only if

$$
\int_{M_{2}} \vec{f} d \sigma_{M_{1}}=0
$$

where $\sigma_{M_{1}}$ is the area element on $M_{1}$.

All conformal maps from $M_{1}$ to $S^{2}$ satisfying the zero mass-center constraint are unique up to the Euclidean rotation group (which is 3 dimensional). We use the Gauss map as the initial condition. 
Definition 4.3. A Gauss map $N: M_{1} \rightarrow S^{2}$ is defined as

$$
N(v)=\vec{n}(v), v \in M_{1},
$$

where $\vec{n}(v)$ is the normal at $v$.

Algorithm 1. Spherical Tuette Mapping

Input (mesh $M$,step length $\delta t$, energy difference threshold $\delta E$ ), output $\left(\vec{t}: M \rightarrow S^{2}\right.$ ) where $\vec{t}$ minimizes the Tuette energy.

1. Compute Gauss map $N: M \rightarrow S^{2}$. Let $\vec{t}=N$, compute Tuette energy $E_{0}$.

2. For each vertex $v \in M$, compute Absolute derivative $D \vec{t}$.

3. Update $\vec{t}(v)$ by $\delta \vec{t}(v)=-D \vec{t}(v) \delta t$.

4. Compute Tuette energy E.

5. If $E-E_{0}<\delta E$, return $\vec{t}$. Otherwise, assign $E$ to $E_{0}$ and repeat steps 2 through to 5 .

Because the Tuette energy has a unique minimum, the algorithm converges rapidly and is stable. We use it as the initial condition for the conformal mapping.

\section{Algorithm 2. Spherical Conformal Mapping}

Input (mesh $M$, step length $\delta$, energy difference threshold $\delta E$ ), output $\left(\vec{h}: M \rightarrow S^{2}\right)$. Here $\vec{h}$ minimizes the harmonic energy and satisfies the zero mass-center constraint.

1. Compute Tuette embedding $\vec{t}$. Let $\vec{h}=\vec{t}$, compute Tuette energy $E_{0}$.

2. For each vertex $v \in M$, compute the absolute derivative $D \vec{h}$.

3. Update $\vec{h}(v)$ by $\delta \vec{h}(v)=-D \vec{h}(v) \delta t$.

4. Compute Möbius transformation $\vec{\varphi}_{0}: S^{2} \rightarrow S^{2}$, such that

$$
\begin{aligned}
\Gamma(\vec{\varphi}) & =\int_{S^{2}} \vec{\varphi} \circ \vec{h} d \sigma_{M_{1}}, \vec{\varphi} \in \operatorname{Mobius}\left(C P^{1}\right) \\
\vec{\varphi}_{0} & =\min _{\vec{\varphi}}\|\Gamma(\vec{\varphi})\|^{2}
\end{aligned}
$$

where $\sigma_{M_{1}}$ is the area element on $M_{1} . \Gamma(\vec{\varphi})$ is the mass center, $\vec{\varphi}$ minimizes the norm in the mass center condition.

5. compute the harmonic energy $E$.

6. If $E-E_{0}<\delta E$, return $\vec{t}$. Otherwise, assign $E$ to $E_{0}$ and repeat step 2 through to step 6 .

Step 4 is non-linear and expensive to compute. In practice we use the following procedure to replace it:

1. Compute the mass center $\vec{c}=\int_{S^{2}} \vec{h} d \sigma_{M_{1}}$;

2. For all $v \in M, \vec{h}(v)=\vec{h}(v)-\vec{c}$;

3. For all $v \in M, \vec{h}(v)=\frac{\vec{h}(v)}{\|\vec{h}(v)\|}$.

This approximation method is good enough for our purpose. By choosing the step length carefully, the energy can be decreased monotonically at each iteration. 


\section{OPTIMIZE THE CONFORMAL PARAMETERIZATION BY LANDMARKS}

In order to compare two brain surfaces, it is desirable to adjust the conformal parameterization and match the geometric features on the brains as well as possible. We define an energy to measure the quality of the parameterization. Suppose two brain surfaces $S_{1}, S_{2}$ are given, conformal parameterizations are denoted as $f_{1}: S^{2} \rightarrow S_{1}$ and $f_{2}: S^{2} \rightarrow S_{2}$, the matching energy is defined as

$$
E\left(f_{1}, f_{2}\right)=\int_{S^{2}}\left\|f_{1}(u, v)-f_{2}(u, v)\right\|^{2} d u d v
$$

We can composite a Möbius transformation $\tau$ with $f_{2}$, such that

$$
E\left(f_{1}, f_{2} \circ \tau\right)=\min _{\zeta \in \Omega} E\left(f_{1}, f_{2} \circ \zeta\right),
$$

where $\Omega$ is the group of Möbius transformations. We use landmarks to obtain the optimal Möbius transformation. Landmarks are commonly used in brain mapping. We manually label the landmarks on the brain as a set of sulcal curves, ${ }^{6}$ as shown in Figure 7. First we conformally map two brains to the sphere, then we pursue an optimal Möbius transformation to minimize the Euclidean distance between the corresponding landmarks on the spheres. Suppose the landmarks are represented as discrete point sets, and denoted as $\left\{p_{i} \in S_{1}\right\}$ and $\left\{q_{i} \in S_{2}\right\}, p_{i}$ matches $q_{i}, i=1,2, \ldots, n$. The landmark mismatch functional for $u \in \Omega$ is defined as

$$
E(u)=\sum_{i=1}^{n}\left\|p_{i}-u\left(q_{i}\right)\right\|^{2}, u \in \Omega, p_{i}, q_{i} \in S^{2}
$$

In general, the above variational problem is a nonlinear one. In order to simplify it, we convert it to a least squares problem. First we project the sphere to the complex plane, then the Möbius transformation is represented as a complex linear rational formula, Equation 14. We add another constraint for $u$, so that $u$ maps infinity to infinity. That means the north poles of the spheres are mapped to each other. Then $u$ can be represented as a linear form $a z+b$. Then the functional of $u$ can be simplified as

$$
E(u)=\sum_{i=1}^{n} g\left(z_{i}\right)\left|a z_{i}+b-\tau_{i}\right|^{2}
$$

where $z_{i}$ is the stereo-projection of $p_{i}, \tau_{i}$ is the projection of $q_{i}, g$ is the conformal factor from the plane to the sphere, it can be simplified as

$$
g(z)=\frac{4}{1+z \bar{z}} .
$$

So the problem is a least squares problem.

\section{SPHERICAL HARMONIC ANALYSIS}

Let $L^{2}\left(S^{2}\right)$ denote the Hilbert space of square integrable functions on the $S^{2}$. In spherical coordinates, $\theta$ is taken as the polar (colatitudinal) coordinate with $\theta \in[0, \pi]$, and $\phi$ as the azimuthal (longitudinal) coordinate with $\phi \in[0,2 \pi)$. The usual inner product is given by

$$
<f, h>=\int_{0}^{\pi}\left[\int_{0}^{2 \pi} f(\theta, \phi) \overline{h(\theta, \phi)} d \phi\right] \sin \theta d \theta .
$$

A function $f: S^{2} \rightarrow \mathbb{R}$ is called a Spherical Harmonic, if it is an eigenfunction of Laplace-Beltrami operator, namely $\Delta f=\lambda f$, where $\lambda$ is a constant. There is a countable set of spherical harmonics which form an orthonormal basis for $L^{2}\left(S^{2}\right)$. 
For any nonnegative integer $l$ and integer $m$ with $|m| \leq l$, the $(l, m)$-spherical harmonic $Y_{l}^{m}$ is a harmonic homogeneous polynomial of degree $l$. The harmonics of degree $l$ span a subspace of $L^{2}\left(S^{2}\right)$ of dimension $2 l+1$ which is invariant under the rotations of the sphere. The expansion of any function $f \in L^{2}\left(S^{2}\right)$ in terms of spherical harmonics can be written

$$
f=\sum_{l \geq 0} \sum_{|m| \leq l} \hat{f}(l, m) Y_{l}^{m}
$$

and $\hat{f}(l, m)$ denotes the $(l, m)$ Fourier coefficient, equal to $\left\langle f, Y_{l}^{m}>\right.$. Spherical harmonic $Y_{l}^{m}$ has an explicit formula

$$
Y_{l}^{m}(\theta, \phi)=k_{l, m} P_{l}^{m}(\cos \theta) e^{i m \phi},
$$

where $P_{l}^{m}$ is the associated Legendre function of degree $l$ and order $m$ and $k_{l, m}$ is a normalization factor. The details are explained in. ${ }^{18}$

Once the brain surface is conformally mapped to $S^{2}$, the surface can be represented as three spherical functions, $x^{0}(\theta, \phi), x^{1}(\theta, \phi)$ and $x^{2}(\theta, \phi)$. The function $x^{i}(\theta, \phi) \in L^{2}\left(S^{2}\right)$ is regularly sampled and transformed to $\hat{x^{i}}(l, m)$ using Fast Spherical Harmonic Transformation as described in. ${ }^{19}$

Many processing tasks that use the geometric surface of the brain can be accomplished in the frequency domain more efficiently, such as geometric compression, matching, surface denoisng, feature detection, and shape analysis. ${ }^{20,21}$

Similar to image compression using Fourier analysis, geometric brain data can be compressed using spherical harmonic analysis. ${ }^{20}$ Global geometric information is concentrated in the low frequency part, whereas noise and locally detailed information is concentrated in the high frequency part. By using low pass filtering, we can keep the major geometric features and compress the brain surface without losing too much information.

\section{EXPERIMENTAL RESULTS}

The 3D brain meshes are reconstructed from 3D 256x256x124 T1 weighted SPGR (spoiled gradient) MRI images, by using an active surface algorithm that deforms a triangulated mesh onto the brain surface. ${ }^{7}$ Figure $3(\mathrm{a})$ and (c) show the same brain scanned at different times. ${ }^{6}$ Because of the inaccuracy introduced by scanner noise in the input data, as well as slight biological changes over time, the geometric information is not exactly the same. Figure 3(a) and (c) reveal minor differences.

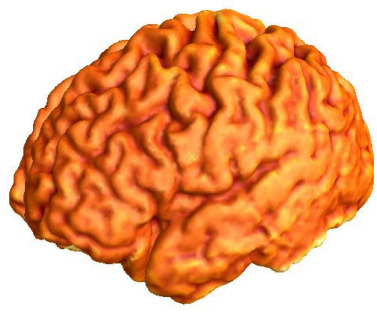

(a)

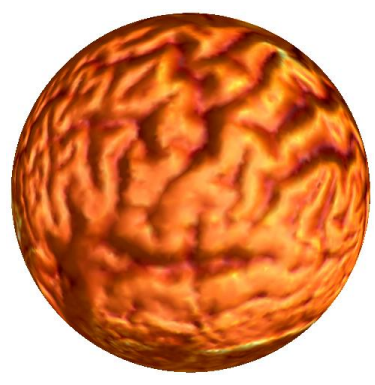

(b)

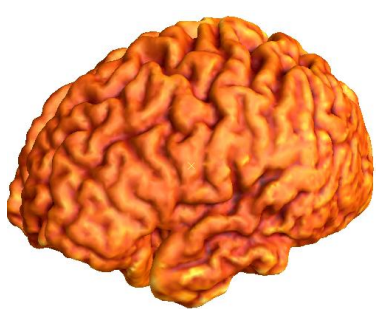

(c)

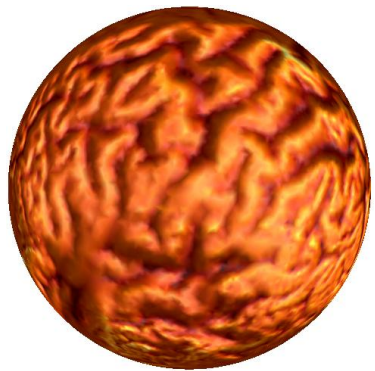

(d)

Figure 3. Reconstructed brain meshes and their spherical harmonic mappings. (a) and (c) are the reconstructed surfaces for the same brain scanned at different times. Due to scanner noise and inaccuracy in the reconstruction algorithm, there are visible geometric differences. (b) and (d) are the spherical conformal mappings of (a) and (c) respectively; the normal information is preserved. By the shading information, the correspondence is illustrated.

The conformal mapping results are shown in Figure 3(b) and (d). From this example, we can see that although the brain meshes are slightly different, the mapping results look quite similar. The major features are mapped to the same position on the sphere. This suggests that the computed conformal mappings continuously depend on the geometry, and can match the major features consistently and reproducibly. In other words, conformal mapping may be a good candidate for a canonical parameterization in brain mapping. 


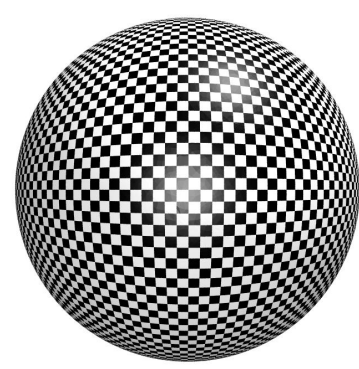

(a)

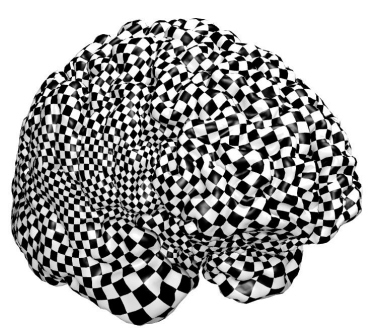

(b)

Figure 4. Conformal texture mapping. (a) Texture mapping of the sphere; (b) Texture mapping of the brain. The conformality is visualized by texture mapping of a checkerboard image. The sphere is mapped to the plane by stereographic projection, then the planar coordinates are used as the texture coordinates. This texture parameter is assigned to the brain surface through the conformal mapping between the sphere and the brain surface. All the right angles on the texture are preserved on the brain surface.

Figure 4 shows the mapping is conformal by texture mapping a checkerboard to both the brain surface mesh and a spherical mesh. Each black or white square in the texture is mapped to sphere by stereographic projection, and pulled back to the brain. Note that the right angles are preserved both on the sphere and the brain.

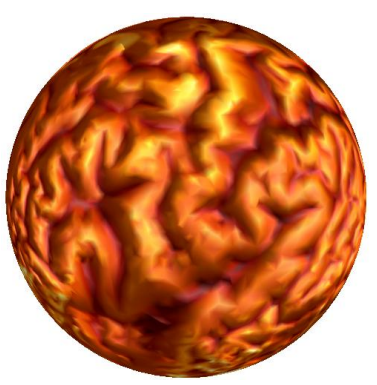

(a)

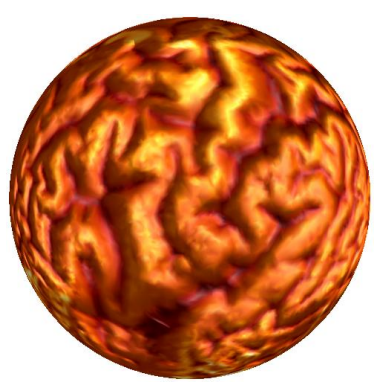

(b)

Figure 5. Conformal mappings of surfaces with different resolutions. (a).Surface with 20,000 faces; (b) Surface with 50,000 faces. The original brain surface has 50,000 faces, and is conformally mapped to a sphere, as shown in (a). Then the brain surface is simplified to 20,000 faces, and its spherical conformal mapping is shown in (b).

Conformal mappings are stable and depend continuously on the input geometry but not on the triangulations, and are insensitive to the resolutions of the data. Figure 5 shows the same surface with different resolutions, and their conformal mappings. The mesh simplification is performed using a standard method. The refined model has 50k faces, coarse one has $20 \mathrm{k}$ faces. The conformal mappings map the major features to the same positions on the spheres.

In order to measure the conformality, we map the iso-polar angle curves and iso-azimuthal angle curves from the sphere to the brain by the inverse conformal mapping, and measure the intersection angles on the brain. The distribution of the angles of a subject(A) are illustrated in Figure 6. The angles are concentrated about the right angle.

Figure 7 shows the landmarks, and the result of the optimization by a Möbius transformation. We also computed the matching energy, following Equation 19. We did our testing on three example subjects. Their information is shown in Table 1. We took subject A as the target brain. For each new subject model, we found a Möbius transformation that minimized the landmark mismatch energy on the maximum intersection subsets of it and A. As shown in Table 1, the matching energies were reduced after the Möbius transformation.

Figure 8 illustrates the geometric compression results using spherical harmonic compression. The low pass filter is applied to remove high frequency components, and the $L^{2}$ error is measured between the reconstructed 


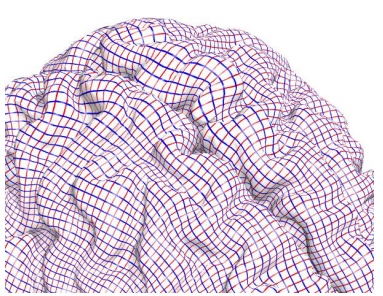

(a)

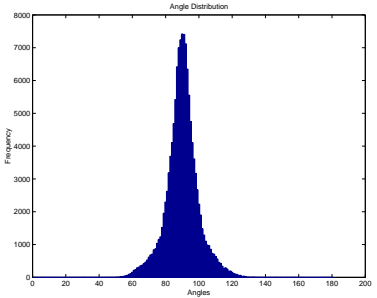

(b)

Figure 6. Conformality measurement. (a) Intersection angles; (b) Angle distribution. The curves of iso-polar angle and iso-azimuthal angle are mapped to the brain, and the intersection angles are measured on the brain. The histogram is illustrated.

\begin{tabular}{|l|c|c|c|c|}
\hline Subject & Vertex \# & Face \# & Before & After \\
\hline A & 65,538 & 131,072 & - & - \\
\hline B & 65,538 & 131,072 & 604.134 & 506.665 \\
\hline C & 65,538 & 131,072 & 414.803 & 365.325 \\
\hline
\end{tabular}

Table 1. Matching energy for three subjects. Subject A was used as the target brain. For subjects B and C, we found Möbius transformations that minimized the landmark mismatch functions, respectively.

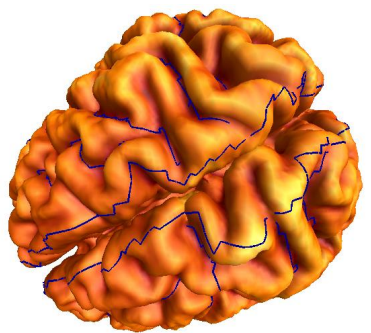

(a)

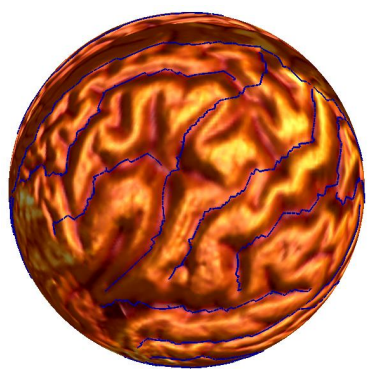

(b)

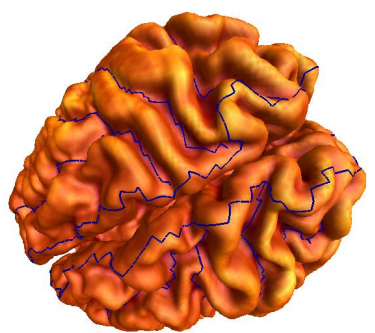

(c)

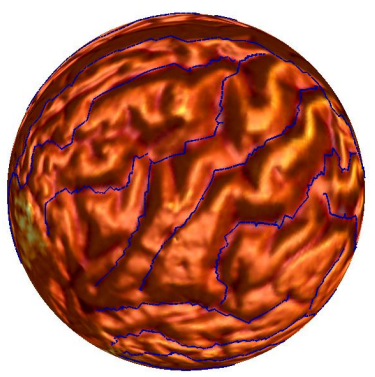

(d)

Figure 7. Möbius transformation to minimize the deviations between landmarks. The blue curves are the landmarks. The correspondence between curves has been preassigned. The desired Möbius transformation is obtained to minimize the matching error on the sphere. 
surface and the original surface.

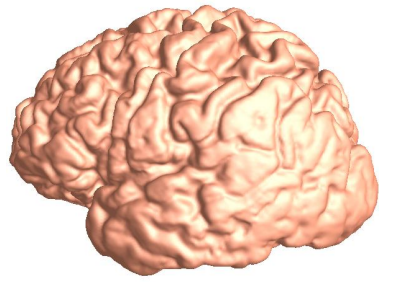

(a)

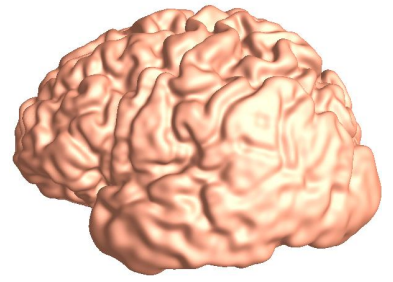

(b)

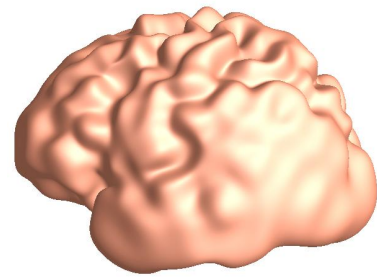

(c)

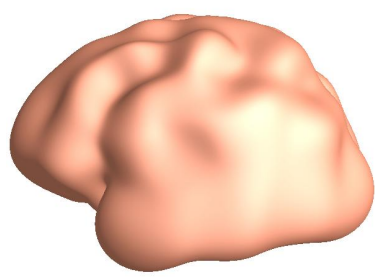

(d)

Figure 8. This figure illustrates the geometry compression results using spherical harmonics. After we conformally map the brain to a sphere, we can use spherical harmonics to compress the geometry. (a) is the original brain surface. (b), (c) and (d) are brain surfaces reconstructed from spherical harmonics with $\frac{1}{8}, \frac{1}{64}$ and $\frac{1}{256}$ of original low frequency coefficients, separately.
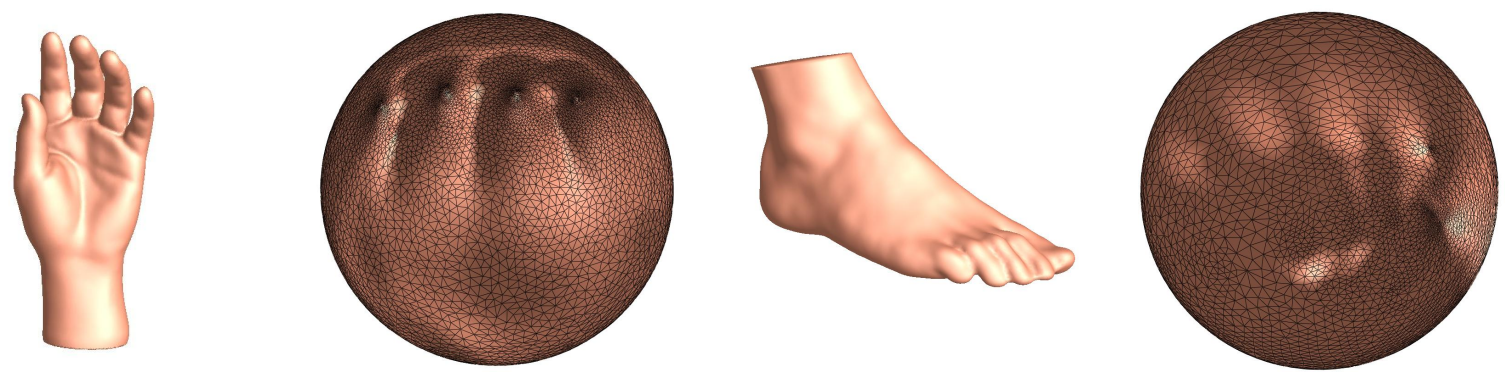

Figure 9. Spherical conformal mapping of genus zero surfaces. Extruding parts (such as fingers and toes) are mapped to denser regions on the sphere.

\section{CONCLUSION AND FUTURE WORK}

In this paper, we apply part of the algorithms ${ }^{1,2}$ (for genus zero) to cortical surface matching problem. The algorithm finds a unique conformal mapping between genus zero manifolds. Our method only depends on the surface geometry and not on the mesh structure (i.e. gridding) and resolution. Our algorithm is very fast and stable in reaching a solution. There are numerous applications of these mapping algorithms, such as providing a canonical space for automated feature identification, brain to brain registration, brain structure segmentation, brain surface denoising, shape analysis and convenient surface visualization, among others. We are trying to generalize this approach to compute conformal mappings between non-zero genus surfaces.

\section{REFERENCES}

1. X. Gu and S. Yau, "Computing conformal structures of surfaces," Communications in Information and Systems 2, pp. 121-146, December 2002.

2. X. Gu and S. Yau, "Global conformal surface parameterization," in ACM Symposium on Geometry Processing, pp. 127-137, 2003.

3. B. Fischl, M. Sereno, R. Tootell, and A. Dale, "High-resolution inter-subject averaging and a coordinate system for the cortical surface," in Human Brain Mapping, 8, pp. 272-284, 1999.

4. M. Hurdal, K. Stephenson, P. Bowers, D. Sumners, and D. Rottenberg, "Coordinate systems for conformal cerebellar flat maps," in NeuroImage, 11, p. S467, 2000. 
5. S. Haker, S. Angenent, A. Tannenbaum, R. Kikinis, G. Sapiro, and M. Halle, "Conformal surface parameterization for texture mapping," IEEE Transactions on Visualization and Computer Graphics 6, pp. 181-189, April-June 2000.

6. P. Thompson, M. Mega, C. Vidal, J. Rapoport, and A. Toga, "Detecting disease-specific patterns of brain structure using cortical pattern matching and a population-based probabilistic brain atlas," in Proc. 17th International Conference on Information Processing in Medical Imaging (IPMI2001), pp. 488-501, (Davis, CA, USA), June 18-22 2001.

7. P. Thompson and A. Toga, "A framework for computational anatomy," in Computing and Visualization in Science, 5, pp. 1-12, 2002.

8. M. Eck, T. DeRose, T. Duchamp, H. Hoppe, M. Lounsbery, and W. Stuetzle, "Multiresolution analysis of arbitrary meshes," in Computer Graphics (Proceedings of SIGGRAPH 95), Auguest 1995.

9. R. Schoen and S. Yau, Lectures on Harmonic Maps, International Press, Harvard University, Cambridge MA, 1997.

10. P. Alliez, M. Meyer, and M. Desbrun, "Interactive geometry remeshing," in Computer Graphics (Proceedings of SIGGRAPH 02), pp. 347-354, 2002.

11. M. Desbrun, M. Meyer, and P. Alliez, "Intrinsic parametrizations of surface meshes," in Proceedings of Eurographics, 2002.

12. U. Pinkall and K. Polthier, "Computing discrete minimal surfaces and their conjugates," in Experim. Math., 2(1), pp. 15-36, 1993.

13. T. Kanai, H. Suzuki, and F. Kimura, "Three-dimensional geometric metamorphosis based on harmonic maps," The Visual Computer 14(4), pp. 166-176, 1998.

14. B. Levy, S. Petitjean, N. Ray, and J. Maillot, "Least squares conformal maps for automatic texture atlas generation," in Computer Graphics (Proceedings of SIGGRAPH 02), Addison Wesley, 2002.

15. S. Angenent, S. Haker, A. Tannenbaum, and R. Kikinis, "Conformal geometry and brain flattening," $M I C$ CAI , pp. 271-278, 1999.

16. A. Sheffer and E. de Sturler, "Parameterization of faceted surfaces for meshing using angle-based flattening," in Engineering with Computers, 17, pp. 326-337, 2001.

17. M. Hurdal, P. Bowers, K. Stephenson, D. Sumners, K. Rehm, K. Schaper, and D. Rottenberg, "Quasiconformally flat mapping the human cerebellum," in Proc. of MICCAI'99, volume 1679 of Lecture Notes in Computer Science, pp. 279-286, Springer-Verlag, 1999.

18. N. Vilenkin, Special Functions and the Theory of Group Representations, American Mathematical Society, Providence, 1968.

19. D. Healy, D. Rockmore, P. Kostelec, and S. Moore, "FFTs for the 2-sphere - improvements and variations," The Journal of Fourier Analysis and Applications 9(4), pp. 341-385, 2003.

20. C. Brechbuhler, G. Gerig, and O. Kubler, "Surface parametrization and shape description," in Visualization Biomed. Comp. 1992, Proc. SPIE 1808, R. Robb, ed., pp. 80-89, 1992.

21. S. Joshi, U. Grenander, and M. Miller, "On the geometry and shape of brain sub-manifolds," International Journal of Pattern Recognition and Artificial Intelligence: Special Issue on Processing of MR Images of the Human 11(8), pp. 1317-1343, 1997. 